\title{
The Evolving Role of Immune Checkpoint Inhibitors in
}

\section{Cancer Treatment}

\author{
Gregory K. Pennock, ${ }^{a}$ Laura Q.M. Chow ${ }^{\mathrm{b}}$

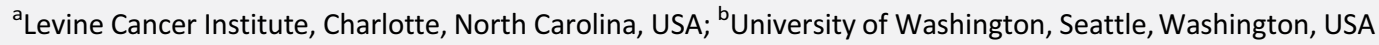 \\ Disclosures of potential conflicts of interest may be found at the end of this article.
}

Key Words. Immunotherapy • Checkpoint inhibitors - CTLA-4 • PD-1 • Combination • Survival

\begin{abstract}
Traditional treatment modalities for advanced cancer (radiotherapy, chemotherapy, or targeted agents) act directly on tumors to inhibit or destroy them. Along with surgery, these modalities are predominantly palliative, with toxicity and only modest improvements in survival in patients with advanced solid tumors. Accordingly, long-term survival rates for most patients with advanced cancer remain low, thus there is a need for cancer treatments with favorable benefit and toxicity profiles that can potentially result in long-term survival. The immune system plays a critical role in the recognition and eradication of tumor cells ("immune surveillance"), and immunotherapies based on this concept have been used for decades with some success against a few tumor types; however, most immunotherapies were limited by a lack of either substantial efficacy or specificity, resulting in toxicity. We

now have a greater understanding of the complex interactions between the immune system and tumors and have identified key molecules that govern these interactions. This information has revitalized the interest in immunotherapy as an evolving treatment modality using immunotherapeutics designed to overcome the mechanisms exploited by tumors to evade immune destruction. Immunotherapies have potentially complementary mechanisms of action that may allow them to be combined with other immunotherapeutics, chemotherapy, targeted therapy, or other traditional therapies. This review discusses the concepts and data behind immunotherapies, with a focus on the checkpoint inhibitors and their responses, toxicities, and potential for long-term survival, and explores promising single-agent and combination therapies in development. The Oncologist 2015;20:812-822
\end{abstract}

Implications for Practice: Immunotherapy is an evolving treatment approach based on the role of the immune system in eradicating cancer. An example of an immunotherapeutic is ipilimumab, an antibody that blocks cytotoxic T-lymphocyte antigen-4 (CTLA-4) to augment antitumor immune responses. Ipilimumab is approved for advanced melanoma and induced long-term survival in a proportion of patients. The programmed death-1 (PD-1) checkpoint inhibitors are promising immunotherapies with demonstrated sustained antitumor responses in several tumors. Because they harness the patient's own immune system, immunotherapies have the potential to be a powerful weapon against cancer.

\section{INTRODUCTION}

Traditional cancer treatments, such as radiotherapy, chemotherapy, and targeted agents, are designed to act directly on tumors by inhibiting their growth and ultimately leading to their destruction. However, resistance mechanisms often develop with agents that have direct effects on tumor cells, and toxicities may limit continued administration at effective levels [1]. As a class of anticancer agents, immunotherapies are designed to harness the patient's own immune system to fight cancer. By directly targeting the immune system, immunotherapies may overcome some of the resistance mechanisms that occur with other agents [2].

More than 100 years ago, Paul Ehrlich was among the first to postulate that the immune system plays a role in recognizing and eradicating tumor cells; it is now well established that tumor cells can actively evade immune destruction to promote tumor growth [3]. Immunotherapies have been used with some success in a few cancer types, for example, interferon- $\alpha$ (IFN- $\alpha$ ) and interleukin-2 in metastatic melanoma and metastatic renal cell carcinoma (RCC) [4]. Evidence of durable responses in a small proportion of treated patients suggests the potential for long-term survival or even a cure $[5,6]$; however, the lack of specificity of these treatments and the potential for serious toxicities has limited their use $[7,8]$.

A greater understanding of the complex interrelationships between the immune system and tumor cells and the mechanisms exploited by tumor cells to evade destruction 
has led to the identification of several specific immunotherapeutic targets [9]. This review provides an overview of this rapidly evolving field, with a focus on immune checkpoint inhibitors as a potential treatment approach for a variety of cancers.

\section{Materials and Methods}

References for this review were identified through searches of PubMed with the search terms "immuno-oncology," "ipilimumab," "cancer," "solid tumors," "immunotherapy," "immune system," "checkpoint receptors," "CTLA-4," and "PD-1." PubMed was last searched in August 2014. Articles were also identified through searches of congress proceedings, the authors' own files, and reference lists from key study papers. The final reference list was generated on the basis of originality and relevance to the scope of this review.

\section{The Immune System and Cancer}

The host immune system has a natural response to cancer, recognizing and eliminating abnormal cells with replicative errors, precancerous cells, and malignant tumor cells from the body. This elimination process has historically been known as "immune surveillance." However, the equilibrium between tumor cells and the immune system can shift in favor of the tumor and can result in uncontrolled malignant growth. This "escape" process can involve the emergence of tumor cells with lower immunogenicity, which dampens the antitumor immune response below the threshold required for complete tumor elimination [3].

The immune response to tumor cells involves both the adaptive and innate components of the immune system. Adaptive antitumor immune responses are mediated by cellular and humoral components, with cytotoxic T lymphocytes (CD4 ${ }^{+}$and $\mathrm{CD} 8^{+}$T cells) (Fig. 1) having a key role. Natural killer (NK) cells have an important role in the innate antitumor immune response [10-13]. Tumor cells exploit multiple complex mechanisms to escape recognition and destruction by the immune system (Fig. 2) [11, 14-20]. As summarized in Table 1, tumor cells can actively dysregulate immune cell activity (notably, $T$ cells and NK cells) [18-22]. This can occur through mechanisms including the activation of T-cell inhibitory (checkpoint) pathways, such as cytotoxic T-lymphocyte antigen 4 (CTLA-4), programmed death-1 (PD-1), and lymphocyte antigen gene 3 (LAG-3); inhibition of T-cell activation pathways (e.g., CD137, OX-40, CD40, GITR, HVEM); and/or suppression of NK cell activity (Fig. 3). Furthermore, the tumor microenvironment contains various immunosuppressive factors from different sources that may be exploited by tumor cells to escape the immune system [11, 14-20].

\section{Immunotherapies}

Counteracting the mechanisms exploited by tumors to suppress and evade the immune system is a rational approach to cancer therapy. A greater understanding of the mechanisms exploited by tumor cells to suppress the immune system and evade destruction has provided a wealth of potential treatment strategies, enabling the development of active immunotherapies that target specific components of the immune system. Alongside the development and evaluation of novel immunotherapeutic agents, translational research efforts have been directed toward trying to understand why some patients respond to immunotherapy and others do not. One

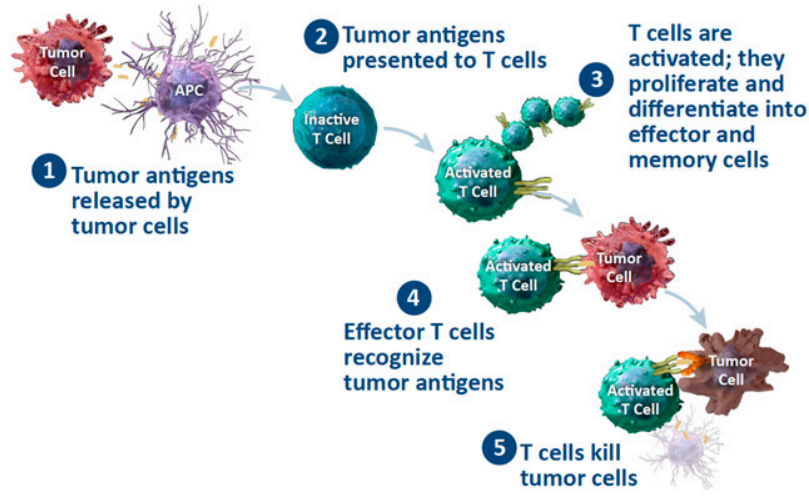

Figure 1. T cells are an important component of the antitumor immune response.

Abbreviation: APC, antigen-presenting cell.

concept is based on the immunologic status of the tumor microenvironment. In some tumors, the tumor microenvironment does not support T-cell infiltration-it is not inflamed and may be less likely to support an antitumor immune response-however, tumors with a T-cell-inflamed microenvironment contain more T cells and thus may be more likely to respond to immunotherapy. Understanding the tumor microenvironment may offer opportunities to predict response to therapy and help physicians select the most appropriate immunotherapy for each patient (Fig. 4) [23]. Another consideration is the mutational heterogeneity of the tumor. Data showed that mutational frequency varies greatly among different tumor types, with melanoma, lung, and bladder cancer having very high mutation rates, whereas rates were low in thyroid cancer, rhabdoid tumors, and acute myeloid leukemia [24]. In theory, the extent of hypermutation within a tumor may predict a better response to immunotherapy because mutation generates neoantigens, which may trigger or support an antitumor immune response.

Understanding the tumor microenvironment may offer opportunities to predict response to therapy and help physicians select the most appropriate immunotherapy for each patient

Vaccines, perhaps the best known example of active immunotherapy, have been widely evaluated for cancer therapy. Most are designed to present specific tumor antigens to the immune system and provide immune modulation to allow the immune response to reach its full potential [25-27]. Although toxicities are low, results from clinical trials have been largely disappointing with limited efficacy, possibly due to trial design or use in the maintenance or adjuvant setting. Sipuleucel-T was approved for asymptomatic or minimally symptomatic metastatic castration-resistant prostate cancer ( $\mathrm{mCRPC}$ ) based on an improvement in median overall survival (OS) versus placebo in a phase III trial [28]. Notably, it is the first and only approved therapeutic cancer vaccine [29].

Another approach under evaluation is adoptive cell transfer of tumor-infiltrating lymphocytes, a passive immunotherapy that has been shown to induce durable complete responses in patients with metastatic melanoma [30]. Because 
Inhibition of tumor antigen presentation

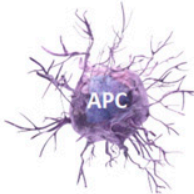

Secretion of immunosuppressive factors

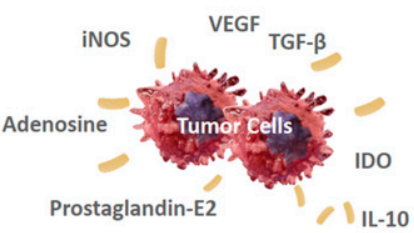

Dysregulation of immune cell activity
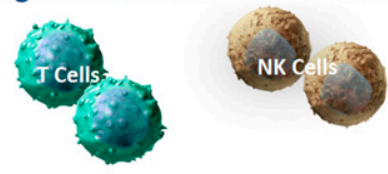

Recruitment of immunosuppressive cell types

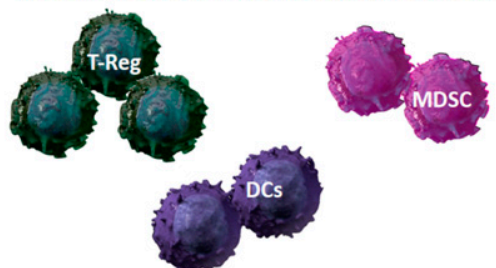

Figure 2. Mechanisms exploited by tumors to evade and suppress the immune system $[11,14-16]$.

Abbreviations: APC, antigen-presenting cell; DC, dendritic cell; IDO, indoleamine 2,3-dioxygenase; iNOS, inducible nitric oxide synthase; MDSC, myeloid-derived suppressor cell; NK, natural killer.

Table 1. Pathways that tumors may exploit to escape the immune system

\begin{tabular}{ll}
\hline Pathway & Role of the tumor and immune effects ${ }^{\text {a }}$ \\
\hline CTLA-4 & $\begin{array}{l}\text { Interaction of CD80/86 on APCs with CTLA-4 on T cells, inhibits T-cell costimulation, } \\
\text { suppressing T-cell activation [18, 21] }\end{array}$ \\
PD-1 & $\begin{array}{l}\text { Expression of PD-1 ligands (PD-L1/PD-L2) by tumor cells binds to PD-1 on T cells and other } \\
\text { immune cells, inhibiting their activity [19] }\end{array}$ \\
$\begin{array}{l}\text { Other T-cell checkpoint and } \\
\text { activation pathways }\end{array}$ & $\begin{array}{l}\text { Tumors may act through a range of direct and indirect mechanisms to dysregulate other } \\
\text { checkpoint and activating pathways involved in T-cell regulation (e.g., LAG-3, TIM-3 }\end{array}$ \\
KIRs & $\begin{array}{l}\text { Ninhibitory]; CD137, OX-40, CD40 [activating]) [18] } \\
\text { that maintain MHCl expression may escape NK cell detection and killing [20] }\end{array}$ \\
FAS/FAS ligand & $\begin{array}{l}\text { Expression of FAS ligand by tumor cells can kill activated T cells expressing FAS (induction of } \\
\text { FAS-mediated cell death; similar role observed for TRAIL/TRAIL ligand) [22] }\end{array}$
\end{tabular}

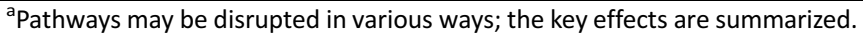

Abbreviations: APCs, antigen-presenting cells; KIRs, killer immunoglobulin-like receptors; $\mathrm{MHCl}$, major histocompatibility complex class 1 ; NK, natural killer; TRAIL, tumor necrosis factor-related apoptosis-inducing ligand.

vaccines and adoptive cell transfer have been widely reviewed elsewhere, they will not be discussed further.

\section{Immunotherapy in the Clinic}

Given the wealth of potential targets to overcome tumorinduced immune suppression, many novel active immunotherapies are in clinical development across a range of solid tumor types (Table 2) [31]. The most advanced in development, including agents approved for patients with advanced melanoma (ipilimumab and pembrolizumab), are checkpoint inhibitors.

\section{Targeting T-Cell Checkpoint Pathways}

T-cell responses are partly regulated through a complex balance of inhibitory ("checkpoint") and activating signals $[10,32]$. Data suggest that tumors may exploit inhibitory checkpoint and activating pathways to suppress T-cell activity, limiting antitumor immune responses. The working hypothesis of immunotherapy focuses on the premise that targeting specific molecules within these pathways can restore the antitumor immune response.

Key targets of immune checkpoint inhibitory pathways include CTLA-4, PD-1, and LAG-3 (Table 1) [18-22]. Ipilimumab, which blocks CTLA-4 to augment antitumor immune responses, was the first checkpoint inhibitor approved for cancer treatment based on its improvements in OS in the treatment of unresectable or metastatic melanoma [2]. Other agents include the anti-CTLA-4 antibody tremelimumab (melanoma), the PD-1 immune checkpoint inhibitors pembrolizumab (MK-3475) and nivolumab, and the PD-L1 targeted agents MPDL3280A (RG7446) and MEDI-4736 [31].

CTLA-4 Immune Checkpoint Pathway. Blockade of CTLA-4 with ipilimumab significantly improved OS in two randomized phase III trials of patients with metastatic melanoma. In the first phase III trial, median OS was 10.1 months with ipilimumab $3 \mathrm{mg} / \mathrm{kg}$ versus 6.4 months with the gp100 vaccine as control $(p<.001)$ [33]. The results formed the basis of the regulatory approval of ipilimumab at $3 \mathrm{mg} / \mathrm{kg}$ in unresectable or metastatic melanoma [2].

In the second phase III trial, ipilimumab $10 \mathrm{mg} / \mathrm{kg}$ plus dacarbazine was compared with placebo plus dacarbazine in first-line treatment. Ipilimumab or placebo was given concurrently with dacarbazine at weeks $1,4,7$, and 10 , followed by dacarbazine alone every 3 weeks through week 22 . Median OS was 11.2 months with ipilimumab versus 9.1 months with placebo $(p<.001)$ [34]. Another anti-CTLA-4 monoclonal antibody, tremelimumab, demonstrated antitumor activity, durable responses, and a similar toxicity profile as ipilimumab but was not approved for advanced melanoma because a 


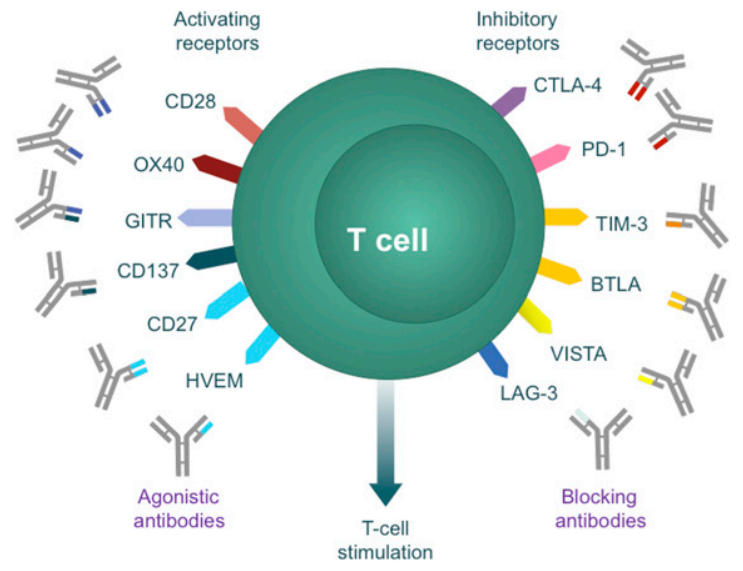

Figure 3. Checkpoint and activating receptors on $\mathrm{T}$ cells are targets for immunotherapy. From [17] with permission from Macmillan Publishers Ltd.

phase III trial failed to show a significant improvement in OS when compared with chemotherapy $[35,36]$.

Ipilimumab is also being evaluated for adjuvant melanoma. Data from a phase III trial of ipilimumab $(n=475)$ versus placebo $(n=476)$ in patients at high risk of relapse (stage IIIA IIIB, or IIIC) showed recurrence-free survival was 26.1 months with ipilimumab versus 17.1 months with placebo (hazard ratio $[\mathrm{HR}]: 0.73 ; p=.0013)$. The incidence of some immune-related adverse events (AEs; e.g., endocrinopathies) was higher in this study [37] than that usually reported in advanced melanoma trials. Another phase III trial evaluating adjuvant ipilimumab compared with high-dose IFN- $\alpha-2 b$ is ongoing (ClinicalTrials.gov identifier NCT01274338) [31].

CTLA-4 inhibition has been evaluated in other solid tumors. Ipilimumab and chemotherapy significantly improved immunerelated progression-free survival (irPFS) and progressionfree survival compared with chemotherapy alone in a phase II study of patients with non-small cell lung cancer (NSCLC) or extensive-disease small cell lung cancer (ED-SCLC) [38, 39]. Immune-related response criteria, discussed later, represent a modification of the Model World Health Organization that was intended to capture the unique tumor response patterns to ipilimumab that include regression of index lesions in the face of new lesions and initial progression, followed by tumor stabilization or a decrease in tumor burden [40]. Median irPFS was 5.7 months with paclitaxel/carboplatin followed by ipilimumab plus paclitaxel/carboplatin (phased regimen: two doses of placebo plus paclitaxel/carboplatin followed by four doses of ipilimumab plus paclitaxel/ carboplatin) versus 4.6 months in NSCLC patients treated with paclitaxel/carboplatin alone. The phased regimen appeared to be superior to the concurrent regimen (ipilimumab plus paclitaxel/carboplatin given concurrently), and on the phased regimen, improvements in irPFS with ipilimumab were greater in patients with squamous compared with nonsquamous histology [38]. In the same trial, median irPFS was 6.4 months with phased ipilimumab/chemotherapy versus 5.3 months with chemotherapy alone in ED-SCLC patients [39].

The combinations of ipilimumab with paclitaxel plus carboplatin for squamous NSCLC and ipilimumab with etoposide plus a platinum agent for SCLC are being evaluated in phase III trials (ClinicalTrials.gov identifiers NCT01285609 and NCT01450761,

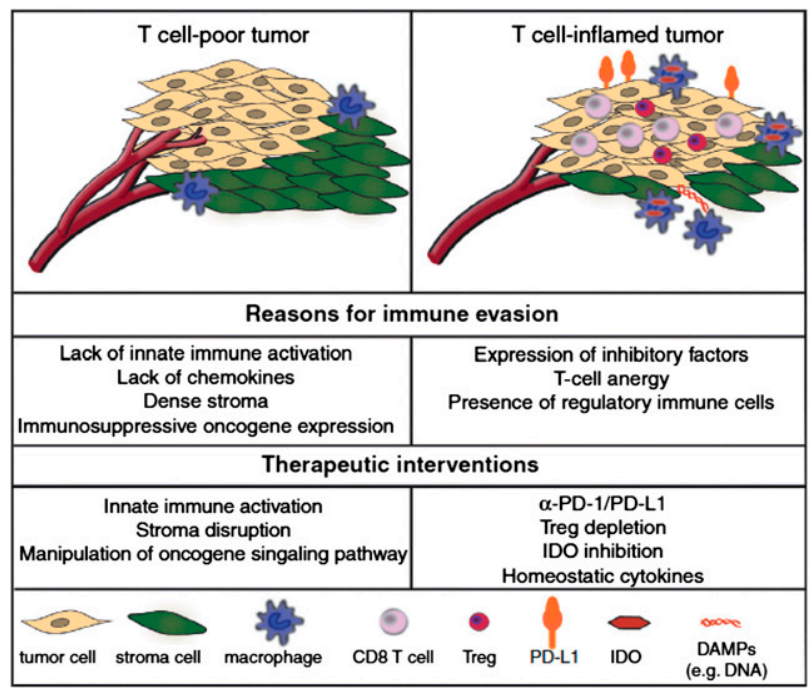

Figure 4. The immune status of the tumor microenvironment may have implications for the selection and success of immunotherapy. From [23] with permission from Elsevier.

Abbreviation: IDO, indoleamine 2,3-dioxygenase.

respectively) [2]. Tremelimumab is also being evaluated in NSCLC in a phase I study in combination with gefitinib in previously treated patients with mutated EGFR (ClinicalTrials.gov identifier NCT02040064) [31].

Ipilimumab and tremelimumab are being evaluated in other solid tumors, including prostate cancer, RCC, pancreatic cancer, mesothelioma, and breast cancer (Table 2) [31]. Data from a phase III trial of single-dose radiotherapy followed by ipilimumab or placebo in previously treated patients with mCRPC showed that the primary endpoint, OS, was not met (ipilimumab vs. placebo, 11.2 vs. 10.0 months, respectively; HR: $0.85 ; p=.053$ ); however, prespecified and exploratory subgroup analyses suggested ipilimumab may have activity in patients with better prognostic features [41]. An ongoing phase III trial in patients with less advanced, chemotherapynaïve $\mathrm{mCRPC}$ will evaluate this hypothesis (ClinicalTrials.gov identifier NCT01057810) [31].

PD-1 Immune Checkpoint Pathway. In contrast to CTLA-4, which regulates de novo immune responses, PD-1 affects ongoing effector immune responses that protect against immune-mediated tissue destruction [9]. Antibodies designed to inhibit the interaction between PD-1 and its ligands (PD-L1 and PD-L2) have shown promising results in clinical trials of different tumor types. Because they have historically responded well to immunotherapies, melanoma and RCC have long been considered "immunogenic" tumors. In contrast, lung cancer, for example, has not historically been considered immunogenic because little success was achieved with immunotherapies (mostly vaccines). Nonetheless, tumor regressions and prolonged responses with improved survival have been observed in early phase trials with PD-1 immune checkpoint inhibitors, supporting the capability of the immune system to recognize and respond to lung cancer and other tumor types not previously considered sensitive to immunotherapy.

Pembrolizumab, a PD-1 immune checkpoint inhibitor, produced a median PFS of $>7$ months in patients with advanced melanoma, some of whom had received prior treatment with ipilimumab [42]. A pooled analysis of 411 patients 
Table 2. Active immunotherapies under evaluation for selected tumors ${ }^{\mathrm{a}}$ (highest development phase only is shown) [31]

\begin{tabular}{|c|c|c|c|c|c|c|c|c|}
\hline Phase & Melanoma & NSCLC/ SCLC & RCC & $\begin{array}{l}\text { Prostate/ } \\
\text { CRPC }\end{array}$ & Pancreatic & $\begin{array}{l}\text { Head and } \\
\text { neck }\end{array}$ & Breast & $\begin{array}{l}\text { Hematologic } \\
\text { malignancies }\end{array}$ \\
\hline \multirow[t]{4}{*}{ III } & $\frac{\text { Anti-CTLA-4 }}{\text { Ipilimumab }}$ & $\frac{\text { Anti-CTLA-4 }}{\text { Ipilimumab }}$ & $\frac{\text { Anti-PD-1 }}{\text { Nivolumab }}$ & $\frac{\text { Anti-CTLA-4 }}{\text { Ipilimumab }}$ & & $\frac{\text { Anti-PD-1 }}{\text { Nivolumab }}$ & & \\
\hline & $\begin{array}{l}\text { Anti-PD-1 } \\
\text { Nivolumab } \\
\text { Pembrolizumab }\end{array}$ & $\begin{array}{l}\text { Anti-PD-1 } \\
\text { Nivolumab } \\
\text { Pembrolizumab }\end{array}$ & & & & & & \\
\hline & $\frac{\text { Anti-PD-L1 }}{\text { MPDL3280A }}$ & $\frac{\text { Anti-PD-L1 }}{\text { MPDL3280A }}$ & & & & & & \\
\hline & & $\frac{\text { Anti-PD-L1 }}{\text { MEDI4736 }}$ & & & & & & \\
\hline \multirow[t]{3}{*}{ II } & $\frac{\text { Anti-PD-1 }}{\text { Pidilizumab }}$ & & $\frac{\text { Anti-PD-L1 }}{\text { MPDL3280A }}$ & $\frac{\text { Anti-CTLA-4 }}{\text { Tremelimumab }}$ & $\frac{\text { Anti-PD-1 }}{\text { Pidilizumab }}$ & & $\frac{\text { IDO inhibitor }}{\text { Indoximod }}$ & $\begin{array}{l}\text { Anti-PD-1 } \\
\text { Nivolumab } \\
\text { Pembrolizumab }\end{array}$ \\
\hline & $\frac{\text { IDO inhibitor }}{\text { INCB024360 }}$ & & & $\frac{\text { Anti-PD-1 }}{\text { Pidilizumab }}$ & & & & \\
\hline & & & & $\frac{\text { IDO inhibitor }}{\text { Indoximod }}$ & & & & \\
\hline \multirow[t]{4}{*}{ I/II } & $\frac{\text { LAG-3 protein }}{\text { IMP321 }}$ & & & $\frac{\text { OX-40 agonist }}{\text { Anti-OX-40 }}$ & $\frac{\text { Anti-CTLA-4 }}{\text { Ipilimumab }}$ & & $\frac{\text { Anti-CTLA-4 }}{\text { Ipilimumab }}$ & $\frac{\text { Anti-PD-1 }}{\text { Pidilizumab }}$ \\
\hline & $\frac{\text { OX-40 agonist }}{\text { Anti-OX-40 }}$ & & & & & & $\frac{\text { Anti-PD-1 }}{\text { Pembrolizumab }^{b}}$ & $\frac{\text { Anti-PD-L1 }}{\text { MPDL3280A }}$ \\
\hline & & & & & & & $\frac{\text { OX-40 agonist }}{\text { Anti-OX-40 }}$ & $\frac{\text { Anti-CTLA-4 }}{\text { Ipilimumab }}$ \\
\hline & & & & & & & & $\frac{\text { CD137 agonist }}{\text { Urelumab }}$ \\
\hline \multirow[t]{6}{*}{ I } & $\frac{\text { Anti-PD-L2 }}{\text { rHIgM12B7 }}$ & rhlL-15 & $\frac{\text { Anti-CTLA-4 }}{\text { Tremelimumab }}$ & $\frac{\text { Anti-PD-L1 }}{\text { MPDL3280A }}$ & $\frac{\text { Anti-CTLA-4 }}{\text { Tremelimumab }}$ & & $\frac{\text { Anti-CTLA-4 }}{\text { Tremelimumab }}$ & $\frac{\text { Anti-LAG-3 }}{\text { BMS-986016 }}$ \\
\hline & $\frac{\text { Anti-PD-L2 }}{\text { rHigM12B7 }}$ & & $\frac{\text { LAG-3 protein }}{\text { IMP321 }}$ & & $\frac{\text { CD40 agonist }}{\text { CP-870,893 }}$ & & $\frac{\text { LAG-3 protein }}{\text { IMP321 }}$ & $\frac{\text { CD137 agonist }}{\text { PF-05082566 }}$ \\
\hline & $\frac{\text { Anti-GITR }}{\text { TRX518 }}$ & & $\underline{\text { rhIL-15 }}$ & & & & & \\
\hline & $\frac{\text { CD40 agonist }}{\text { CP-870,893 }}$ & & & & & & & \\
\hline & $\begin{array}{l}\text { GITR agonist } \\
\text { TRX518 }\end{array}$ & & & & & & & \\
\hline & rhIL-15 & & & & & & & \\
\hline
\end{tabular}

showed a 1-year OS rate of $71 \%$ and a manageable safety profile [43]. Pembrolizumab was the first PD-1 immune checkpoint inhibitor to be approved by the U.S. Food and Drug Administration (FDA); it was approved for the treatment of patients with unresectable or metastatic melanoma and disease progression following ipilimumab, and, if BRAF V600 mutation positive, a BRAF inhibitor. Initial data from a phase I trial of pembrolizumab in previously treated and untreated patients with advanced NSCLC showed evidence of robust antitumor activity and agent tolerability $[44,45]$. In a phase Ib trial of pembrolizumab in selected advanced solid malignancies, early data from patients with human papillomavirusassociated (HPV-associated) and non-HPV-associated head and neck cancer $(n=59)$ showed an encouraging response rate of $20 \%$ (confirmed and unconfirmed responses) and stable disease in $29 \%$ of patients. Pembrolizumab was well tolerated overall, with pruritus (10\%), fatigue (7\%), and rash (7\%) as the most frequently reported drug-related AEs [46]. This trial continues, evaluating pembrolizumab in malignancies, including triple-negative breast cancer, urothelial cancer, and gastric cancer (ClinicalTrials.gov identifier NCT01848834). Ongoing phase III clinical trials will provide further information about pembrolizumab in melanoma (ClinicalTrials.gov identifier NCT01866319), NSCLC (ClinicalTrials.gov identifier NCT01905657), and squamous cell cancer of the head and neck (ClinicalTrials.gov identifier NCT02252042) [31].

Another PD-1 immune checkpoint inhibitor, nivolumab, achieved sustained OS in patients with advanced solid tumors, including NSCLC, melanoma, and RCC in an early phase clinical trial. Median OS was 9.6 months, 16.8 months, and $>22$ months in patients with NSCLC, melanoma, and RCC, respectively [47]. A large phase I trial (CheckMate 012, ClinicalTrials.gov identifier NCT01454102) is in progress in chemotherapy-naïve patients with NSCLC to evaluate nivolumab as monotherapy and in a range of combinations, including with ipilimumab, in three-platinum-based doublet chemotherapy regimens, with bevacizumab given after at least 4 cycles of platinum doublet chemotherapy, and with erlotinib in patients 
with EGFR mutation-positive nonsquamous NSCLC. Preliminary data showed activity with the various chemotherapy combination regimens [48]; objective responses occurred in $33 \%-47 \%$ of patients across the treatment arms. Similarly, initial data with the erlotinib and ipilimumab combinations showed the combinations were feasible and provided evidence of durable clinical activity despite some increase in toxicity $[49,50]$. In 2014, nivolumab was approved in Japan for patients with unresectable melanoma, and the FDA recently approved it for patients with unresectable or advanced melanoma who no longer respond to other drugs. Nivolumab is being investigated in phase II/III trials in advanced NSCLC (ClinicalTrials.gov identifiers NCT02041533, NCT02066636, NCT01642004, and NCT01673867), RCC (ClinicalTrials.gov identifier NCT01668784), melanoma (ClinicalTrials.govidentifiers NCT01721772 and NCT01721746), and head and neck cancer (ClinicalTrials.gov identifier NCT02105636) [31]. Encouraging data have also been reported from phase I trials with nivolumab in patients with relapsed or refractory lymphoid malignancies [51, 52]. Several phase I and II trials are ongoing to evaluate nivolumab further in patients with hematologic malignancies (e.g., ClinicalTrials.gov identifiers NCT02275533, NCT02038946, NCT02038933, and NCT02181738) [31].

Pidilizumab is an anti-PD-1 monoclonal antibody. Data from a phase II trial of 103 patients with metastatic melanoma, including 51\% who had previously received ipilimumab, showed a 1-year OS rate of $64.5 \%$ [53]. Other trials with this agent are in progress (ClinicalTrials.gov identifier NCT01952769 and NCT02077959) [31]. MEDI-0680 (AMP514 ) is a PD-1 inhibitor under evaluation in a phase I trial (ClinicalTrials.gov identifier NCT02013804) [31].

The anti-PD-L1 antibody MPDL3280A (RG7446) has been evaluated in a phase I expansion study in patients with squamous or nonsquamous NSCLC, and 37 NSCLC patients were evaluable for efficacy [54]. An objective response rate (ORR) of $24 \%$ (9 of 37 ) was observed with a duration of response in the range of $>1$ to $>214$ days, and the 24 -week PFS was $46 \%$. Additional patients had delayed responses after apparent radiographic progression (not included in the ORR). A phase III trial is ongoing (ClinicalTrials.gov identifier NCT02008227). Data from a cohort of patients with metastatic urothelial bladder cancer ( $n=30$ with a minimum of 6 weeks of follow-up) in a phase I trial showed an ORR of $43 \%$ with grade $3 / 4$ drug-related AEs in $4.4 \%$ of patients $(n=68)$ [55]. A phase II trial is ongoing in patients with locally advanced or metastatic urothelial bladder cancer (ClinicalTrials.gov identifier NCT02108652) [31]. MEDI4736, another anti-PD-L1 monoclonal antibody, is under evaluation in NSCLC including a phase III trial as sequential therapy for patients who have not progressed following platinum-based concurrent chemoradiation therapy (ClinicalTrials.gov identifier NCT02125461) and a phase II trial in patients with stage IIIB-IV disease who have received at least two prior systemic regimens (ClinicalTrials.gov identifier NCT02087423) [31].

Several other agents designed to inhibit the PD-1 pathway are in early phase development, including the anti-PD-L2 monoclonal antibodies rHIgM12B7 (ClinicalTrials.gov identifier NCT00658892) and AMP-224 (ClinicalTrials.gov identifier NCT01352884) (Table 2) [31]. At present, the immature clinical data and lack of comparative clinical trials means the activity and safety of the different PD-1-pathway targeted agents cannot be compared directly. The different roles of the receptor and ligands in the immune system suggest that there is the potential for agents targeting either the receptor or a ligand (PD-L1 or PD-L2) to have different clinical profiles. It has been proposed that agents inhibiting PD-1 may be more active than PD-L1-directed agents because they can inhibit binding to both PD-L1 and PD-L2, targeting tumors that express higher levels of both receptors; however, this broader inhibition might also be associated with increased inflammatory toxicity [56].

Alongside evaluating the efficacy and safety of these agents, PD-L1 expression is being evaluated as a biomarker of response. Evidence suggests that tumor PD-L1 expression may be associated with response, although responses have been observed in patients with no or low PD-L1 expression $[57,58]$. An analysis of responses to pembrolizumab treatment in patients with advanced melanoma showed a response rate of $51 \%$ of patients with PD-L1-positive tumors versus $6 \%$ in patients with PD-L1-negative tumors [58]. Similarly, in a phase III trial of nivolumab versus chemotherapy in previously untreated patients with advanced melanoma, the response rate to nivolumab was $52.7 \%$ in patients with PD-L1-positive tumors compared with $33.1 \%$ in patients with PD-L1-negative or indiscriminate tumors [59]. An analysis of responses to MPDL3280A showed that across a range of solid tumor types, responses were observed in patients with high PD-L1 tumor expression, especially when PD-L1 was expressed by tumorinfiltrating immune cells [60]. Further evaluation is needed to define fully the predictive value of PD-L1 expression.

Evidence suggests that tumor PD-L1 expression may be associated with response, although responses have been observed in patients with no or low PD-L1 expression. An analysis of responses to pembrolizumab treatment in patients with advanced melanoma showed a response rate of $51 \%$ of patients with PD-L1-positive tumors versus $6 \%$ in patients with PD-L1-negative tumors.

LAG-3 Checkpoint Pathway. LAG-3 (CD223) is an immune checkpoint pathway that can regulate T-cell activity, particularly via its role in enhancing the function of regulatory T cells [18]. IMP321, a LAG-3 immunoglobulin fusion protein, has been evaluated in phase $\mathrm{I} / \mathrm{II}$ trials in a range of solid tumors, either alone or in combination with chemotherapy. Combination therapy with paclitaxel and IMP321 achieved clinical benefit in $90 \%$ of patients with metastatic breast cancer (ORR 50\%) [61]. IMP321 has also shown evidence of activity in a preliminary study of patients with advanced RCC [62], and it is being evaluated in a phase I/II trial in stage II-IV melanoma patients with HLA-A2 genotype (ClinicalTrials.gov identifier NCT01308294) [31]. BMS-986016, an anti-LAG-3 monoclonal antibody, is in phase I development for patients with advanced solid tumors, alone and in combination with nivolumab (ClinicalTrials.gov identifier NCT01968109) [31].

\section{T-Cell-Activating Pathways}

Several T-cell costimulatory receptors are potential targets for active immunotherapy, including CD137, OX-40, CD40, and 
glucocorticoid-induced tumor necrosis factor receptor-related gene (GITR). Agents targeting these costimulatory receptors are shown in Table 2 [31] and include urelumab and PF-05082566, both agonistic CD137 monoclonal antibodies $[63,64]$; TRX518, an agonist GITR monoclonal antibody [31]; an agonistic OX-40 monoclonal antibody [65]; and CP-870,893, an agonistic CD40 monoclonal antibody [66].

Other active immunotherapies include agents designed to manipulate the activation of NK cell inhibitory receptors (killercell Ig-like receptors [KIRs]) [20,67-69] or inhibit indoleamine 2,3-dioxygenase [70, 71]. Several phase I trials are in progress to evaluate the use of PD-1 checkpoint inhibitors in combination with these agents (e.g., ClinicalTrials.gov identifiers NCT01714739 and NCT02178722) [31].

\section{Long-Term Survival Benefit}

Because the checkpoint inhibitors discussed are designed to promote the patient's own antitumor immune response, given the natural adaptability of the immune system response and its capacity to develop durable immune memory, it is conceivable that these therapies will have the potential for long-term survival. Evidence for a long-term survival benefit has been provided by follow-up survival analyses of patients with advanced melanoma who received ipilimumab in phase II and III studies. In the registrational phase III trial (MDX010-20) in previously treated patients with advanced melanoma, 2- and 3-year survival rates were 25\% [72]. In the second phase III trial, a near doubling of the 5-year survival rate was observed with ipilimumab plus dacarbazine compared with placebo plus dacarbazine (18.2\% vs. 8.8\%) [73]. In a pooled analysis of data from 12 ipilimumab clinical trials in advanced melanoma with follow-up to 10 years in some patients, a plateau in the OS curve began at approximately 3 years, and the 3 -year survival rate was $22 \%$ (Fig. 5) [74].

In patients with melanoma, NSCLC, and RCC, preliminary data from phase I studies indicated that patients can achieve durable outcomes after treatment with the PD-1 inhibitors nivolumab and pembrolizumab [42, 43, 47]. Patients with $\operatorname{NSCLC}(n=129)$ and RCC $(n=34)$ treated with nivolumab demonstrated 1- and 2-year OS rates of $42 \%$ and $22 \%$ ( $56 \%$ and $45 \%$ at $3 \mathrm{mg} / \mathrm{kg}$ only; $n=37$ ) and $70 \%$ and $50 \%$, respectively $[47,75]$. In patients with melanoma $(n=107), 1-, 2-$, and 3-year OS rates of $63 \%, 48 \%$, and $41 \%$, respectively, have been reported [76]. In the phase I trial with pembrolizumab in melanoma, responses appeared durable, with $88 \%$ ongoing at the time of analysis [43]. Similarly, in patients with NSCLC, pembrolizumab responses appeared durable, and 1-year survival rates were encouraging $[44,45]$. Furthermore, the manageable tolerability profiles and the relatively low incidence of toxicities (described in "AE Profile") combined with the potential for durable survival makes these PD-1 pathwaydirected agents particularly exciting prospects for the future.

\section{AE Profile}

It is now well established that checkpoint inhibitors are associated with a unique AE profile $[2,77,78]$. These AEs are inflammatory in nature, consistent with increased immune activity, and reflect the mechanism of action of these therapies. Accordingly, they have been termed "immune-related" AEs (irAEs) in clinical studies of ipilimumab [2].

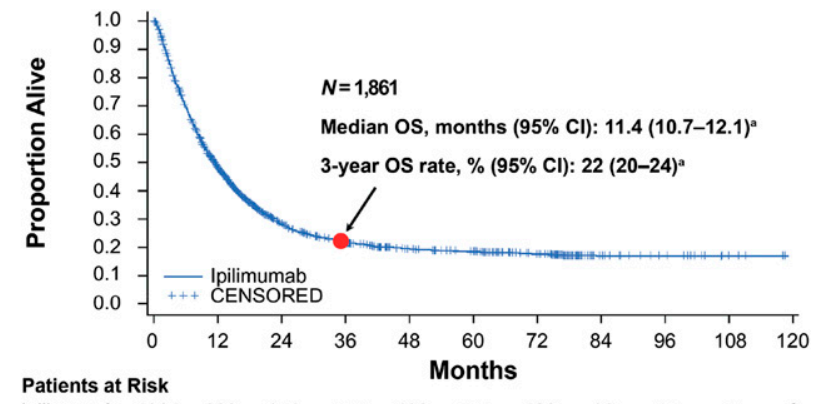

$\begin{array}{llllllllllll}\text { Ipilimumab } & 1861 & 839 & 370 & 254 & 192 & 170 & 120 & 26 & 15 & 5 & 0\end{array}$

${ }^{a}$ Ipilimumab was given at different doses and lines of therapy, and using different schedules across the 12 studies.

Figure 5. Long-term survival in melanoma patients treated with ipilimumab: results of a pooled analysis. From [74] with permission from the American Society of Clinical Oncology.

Abbreviations: $\mathrm{Cl}$, confidence interval; OS, overall survival.

Some irAEs may present like AEs seen with other anticancer therapies, but they have different etiologies and require different management strategies. In clinical trials of ipilimumab, protocol-specific treatment guidelines were developed to help the investigators manage inflammatory AEs [2, 79-81]. Because irAEs can be serious and potentially fatal (e.g., colitis leading to intestinal perforation), prompt recognition and treatment with systemic corticosteroids are required to minimize life-threatening complications. Education of both the patient and the health care team regarding irAEs and their management is critical [82].

The organ systems often affected by checkpoint inhibitors include the skin, the endocrine system, the liver, the gastrointestinal tract, the nervous system, the eyes, the respiratory system, and the hematopoietic system $[2,77,78]$. Frequently reported drug-related AEs with ipilimumab, nivolumab, and pembrolizumab are summarized in Table 3 $[33,42,83]$. Inhibition of CTLA-4 induces well-documented irAEs, including colitis/diarrhea, dermatitis, hepatitis, and endocrinopathies, including uveitis, nephritis, and inflammatory myopathy $[79,80]$. Common treatment-associated AEs with the PD-1 inhibitors nivolumab and pembrolizumab include fatigue, rash, and diarrhea (Table 3) [33, 42, 83]. Immune-mediated pneumonitis has been reported in patients treated with nivolumab and pembrolizumab $[42,83]$. This may be a concern for additional morbidity and mortality in patients with NSCLC who already have compromised lung function. Although there is no data yet from trials designed to compare CTLA-4 and PD-1 checkpoint inhibitors directly, data from separate trials suggest PD-1 inhibitors may be associated with a lower incidence of AEs. Overall, awareness of the unique $A E$ profiles of immunotherapies and their proper management will be key to maximizing the clinical benefit of immunotherapies.

\section{The Potential of Combination Therapy}

It is rational to consider that active immunotherapies may be most effective when used in concurrent or sequential combination with immunotherapies that have different but potentially complimentary mechanisms of action. Initial support for a synergistic effect has come from a phase I study of dual T-cell checkpoint inhibition with ipilimumab and nivolumab in patients with advanced stage III or IV melanoma 
Table 3. Overview of treatment-related AEs reported in trials with select immunotherapies

\begin{tabular}{|c|c|c|c|}
\hline Agent & Ipilimumab & Nivolumab & Pembrolizumab \\
\hline Trial & $\begin{array}{l}\text { Phase III trial in patients with } \\
\text { metastatic melanoma }(n=131 \\
\text { ipilimumab alone arm) [33] }\end{array}$ & $\begin{array}{l}\text { Phase I trial in patients } \\
\text { with advanced solid } \\
\text { tumors }(N=296)[83]\end{array}$ & $\begin{array}{l}\text { Phase I trial in patients } \\
\text { with advanced } \\
\text { melanoma }(N=135)[42]\end{array}$ \\
\hline All treatment-related AEs & 80.2 & 70 & 79 \\
\hline $\begin{array}{l}\text { All grade } 3-4 \\
\text { treatment-related AEs }\end{array}$ & 22.9 & 14 & 13 \\
\hline \multirow{4}{*}{$\begin{array}{l}\text { Most frequently reported } \\
\text { treatment-related AEs; } \\
\text { incidence (grade } 3-4 \text { incidence) }\end{array}$} & Fatigue; 42 (6.9) & Fatigue; 24 (2) & Fatigue; $30,(1)$ \\
\hline & Nausea; 35.1 (2.3) & Rash; 12 (0) & Rash; 21 (2) \\
\hline & Diarrhea; 32.8 (5.3) & Diarrhea; 11 (1) & Pruritus; 21 (1) \\
\hline & Decreased appetite; 26.7 (1.5) & Pruritus; $10(0.3)$ & Diarrhea; 20 (1) \\
\hline All immune-related $A E s^{b}$ & 61.1 & 41 & N/A \\
\hline All grade 3-4 immune-related AEs & 14.5 & 6 & N/A \\
\hline \multirow{4}{*}{$\begin{array}{l}\text { Most frequently reported } \\
\text { immune-related AEs incidence } \\
\text { (grade 3-4 incidence) }^{\mathrm{a}}\end{array}$} & Pruritus; $24.4(0)$ & Rash; 12 (0) & \multirow[t]{4}{*}{ N/A } \\
\hline & Diarrhea; 27.5 (4.6) & Diarrhea; 11 (1) & \\
\hline & Rash; 19.1 (0.8) & Pruritus; $9(<1)$ & \\
\hline & Colitis; 7.6 (5.3) & $\begin{array}{l}\text { Increased alanine } \\
\text { aminotransferase; } 4 \text { (1) }\end{array}$ & \\
\hline
\end{tabular}

All data are shown as percentages.

${ }^{a}$ Four most frequently reported AEs for each agent are shown.

${ }^{b}$ Also termed $A E s$ of special interest.

Abbreviations: $A E$, adverse event; N/A, not available in detailed format (narrative provided for selected AEs only).

[84]. Patients were treated with both ipilimumab (1 or $3 \mathrm{mg} / \mathrm{kg}$ ) and nivolumab $(0.3,1$, or $3 \mathrm{mg} / \mathrm{kg})$ in a concurrent or sequenced regimen. The 2-year OS rate across concurrent dose cohorts ( $n=53$ ) was $79 \%$. In the 17 patients who received nivolumab $1 \mathrm{mg} / \mathrm{kg}$ plus ipilimumab $3 \mathrm{mg} / \mathrm{kg}$, the 1 - and 2-year OS rates were $94 \%$ and $88 \%$, respectively [85]. The types of AEs occurring in patients treated with the combination were similar to previous experience with monotherapy, but the frequency appeared to be higher: $96 \%$ of patients receiving the concurrent regimen experienced a treatment-related $\mathrm{AE}$, and $65 \%$ experienced a grade 3 or 4 event. Twenty-two percent of patients discontinued because of a treatment-related $A E$ $[84,85]$. Interim data with this combination in patients with NSCLC also suggested an increased frequency of AEs [50]. These data emphasize the care required when combining immunotherapies with each other or existing treatment modalities because of the potential for unpredictable toxicity.

The combination of ipilimumab and nivolumab is being evaluated further in a phase III study (CheckMate 067) in which ipilimumab/nivolumab are given concurrently in patients with previously untreated, unresectable, or metastatic melanoma (ClinicalTrials.gov identifier NCT01844505). Interestingly, the results of the phase I study showed that patients who were previously treated with ipilimumab and did not respond could achieve objective responses on subsequent treatment with nivolumab. This suggests that the combination or sequencing of immune checkpoint inhibitors may be used to restore activity in patients who have progressed on one inhibitor. This strategy may also offer a more powerful tool to induce immune activity in tumors that respond poorly, (i.e., those that may have a greater level of immune suppression). Others trials are also in progress evaluating checkpoint inhibitor combinations, for example, an anti-PD-1 antibody (MEDI-0680) with an anti-PD-L1 antibody (MEDI-4736) in patients with advanced malignancies (ClinicalTrials.gov identifier NCT02118337), nivolumab plus urelumab (ClinicalTrials.gov identifier NCT02253992), and pembrolizumab plus PF-05082566 (ClinicalTrials.gov identifier NCT02179918) [31].

Immunotherapies offer the possibility of use in concurrent or sequential combination with chemotherapy, radiotherapy, and targeted therapies. These modalities have various immunepotentiating activities in addition to their cytotoxic/cytostatic effects, including modification of the tumor microenvironment to reduce immune suppression and the release of tumor antigens [86-92]. Particularly interesting are the reports of a potential immune-mediated abscopal effect when radiotherapy is combined with immunotherapy [93]; however, clinical data indicate that the potential for enhanced activity may come with the potential for enhanced toxicity, highlighting the importance of determining the optimal dose, schedule, and sequence when combining an immunotherapy with another treatment modality [34, 38, 39, 94-97].

\section{Clinical Profile: Response Patterns}

Because the mechanism of action of checkpoint inhibitors differs from other treatment modalities, it is not unexpected that their response patterns will also differ [40, 98, 99].

Four distinct patterns of response have been observed with ipilimumab monotherapy [40, 98, 100]: (a) shrinkage in baseline lesions, without new lesions (similar to chemotherapy); (b) durable stable disease (in some patients followed by a slow, steady decline in total tumor burden); (c) response after an increase in total tumor burden (pseudoprogression); and (d) response in the presence of new lesions. The first two patterns could be captured by standard response criteria (World Health Organization criteria or Response Evaluation Criteria In Solid Tumors), whereas the other two would be classified as progressive disease. Novel criteria (immune-related response criteria) have been developed that capture all four potential response patterns [40]. 


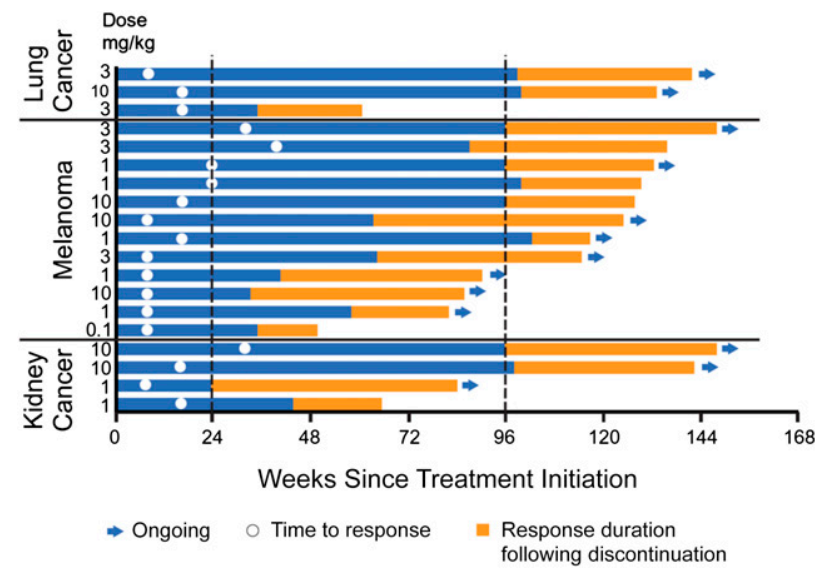

Figure 6. Response duration with nivolumab in a phase I trial: responses may occur early or late, and may continue after discontinuation. From [47] with permission from Dr. F.S. Hodi, Dana-Farber Cancer Institute, Boston, MA.

Similar, unconventional patterns of response have been observed with nivolumab, pembrolizumab, and MPDL3280A $[99,101,102]$. Figure 6 depicts the variable responses seen in a phase I trial with nivolumab $[47,83]$. Responses could occur early or late and continue after discontinuation (patients could be treated up to 96 weeks).

Clinicians need to be aware of the potential for these unconventional response patterns, particularly the possibility of delayed or late responses, so that treatment is not stopped early in patients who may be benefiting. In clinical studies of ipilimumab in patients with advanced melanoma, delayed responses and improvements in survival were observed in patients with stable disease or early immune-related progression $[2,40]$. These findings suggest that the ORR may underestimate the treatment effect of immunotherapies and that OS remains the best endpoint to fully capture the activity of these agents.

Clinicians need to be aware of the potential for these unconventional response patterns, particularly the possibility of delayed or late responses, so that treatment is not stopped early in patients who may be benefiting.

\section{CONCLUSION}

Immunotherapy offers a rational approach to counter the mechanisms tumors exploit to evade destruction by the immune system. In contrast to other therapeutic modalities, an approach designed to harness the patient's own immune system has the potential for benefits, including activity across a broad range of tumor types and durable, long-term survival. In particular, the CTLA-4 and PD-1 immune checkpoint inhibitors offer the opportunity of tumor regression and prolonged survival in solid tumor types previously considered unlikely to respond to immunotherapy. Clinical experience with these agents has shown that an early diagnosis and appropriate management of irAEs can minimize serious complications. Health care providers also need to be aware of the potential for unconventional response patterns when making treatment decisions to avoid premature termination of treatment in patients who may be benefiting. Although agents are progressing quickly from phase I trials to approval, there is still a lot to understand, such as the most accurate endpoints to use and optimal response criteria. We also need to define the best scheduling and combination regimens and associated toxicity profiles and their management. Given the encouraging data, opportunities for combination or sequential approaches, and additional targets yet to be investigated, immunotherapies may become the foundation of cancer therapy in the future, offering long-lasting responses, improvements in survival, and the potential for long-term remission or even a cure for cancer.

\section{ACKNOWLEDGMENTS}

The authors take full responsibility for the content of this publication and confirm that it reflects their viewpoint and medical expertise. Professional medical writing and editorial assistance was provided by Rebecca Turner and Karin McGlynn at StemScientific, an Ashfield Company, and was funded by Bristol-Myers Squibb. Neither Bristol-Myers Squibb nor StemScientific influenced the content of the manuscript. The authors received no financial compensation for authoring the manuscript.

\author{
Author Contributions \\ Conception/Design: Gregory K. Pennock, Laura Q.M. Chow \\ Collection and/or assembly of data: Gregory K. Pennock \\ Data analysis and interpretation: Gregory K. Pennock \\ Manuscript writing: Laura Q.M. Chow \\ Final approval of manuscript: Gregory K. Pennock, Laura Q.M. Chow
}

\section{Disclosures}

Gregory K. Pennock: Bristol-Myers Squibb (H); Laura Q.M. Chow: Amgen, Merck, Novartis (C/A), Genentech, Bristol-Myers Squibb, OSI Pharmaceuticals, AstraZeneca, Novartis, VentiRx, Imclone, Merck, Pfizer (RF).

(C/A) Consulting/advisory relationship; (RF) Research funding; (E) Employment; (ET) Expert testimony; (H) Honoraria received; (OI) Ownership interests; (IP) Intellectual property rights/ inventor/patent holder; (SAB) Scientific advisory board

\section{REFERENCES}

1. Lovly CM, Shaw AT. Molecular pathways: Resistance to kinase inhibitors and implications for therapeutic strategies. Clin Cancer Res 2014;20: 2249-2256.

2. Wolchok JD, Hodi FS, Weber JS et al Development of ipilimumab: A novel immunotherapeutic approach for the treatment of advanced melanoma. Ann N Y Acad Sci 2013;1291:1-13.

3. Dunn GP, Bruce AT, Ikeda $\mathrm{H}$ et al. Cancer immunoediting: From immunosurveillance to tumor escape. Nat Immunol 2002;3:991-998.
4. Waldmann TA. Immunotherapy: Past, present and future. Nat Med 2003;9:269-277.

5. Rosenberg SA, Yang JC, White DE et al. Durability of complete responses in patients with metastatic cancer treated with high-dose interleukin-2: Identification of the antigens mediating response. Ann Surg 1998;228:307319.

6. Rosenberg SA. Raising the bar: The curative potential of human cancer immunotherapy. Sci Transl Med 2012;4:127ps8.
7. Amin A, White RLJr. High-dose interleukin-2: Is it still indicated for melanoma and RCC in an era of targeted therapies? Oncology (Williston Park) 2013;27:680-691.

8. Antony GK, Dudek AZ. Interleukin 2 in cancer therapy. Curr Med Chem 2010;17:3297-3302.

9. Topalian SL, Weiner GJ, Pardoll DM. Cancer immunotherapy comes of age. J Clin Oncol 2011;29: 4828-4836.

10. Finn OJ. Immuno-oncology: Understanding the function and dysfunction of the immune system in cancer. Ann Oncol 2012;23:viii6-viii9. 
11. Vesely MD, Kershaw MH, Schreiber RD et al. Natural innate and adaptive immunity to cancer. Annu Rev Immunol 2011;29:235-271.

12. Godet $Y$, Fabre $E$, Dosset $M$ et al. Analysis of spontaneous tumor-specific CD4 T-cell immunity in lung cancer using promiscuous HLA-DR telomerasederived epitopes: Potential synergistic effect with chemotherapy response. Clin Cancer Res 2012;18: 2943-2953.

13. Mlecnik B, Bindea $G$, Pagès $F$ et al. Tumor immunosurveillance in human cancers. Cancer Metastasis Rev 2011;30:5-12.

14. Drake CG, Jaffee E, Pardoll DM. Mechanisms of immune evasion by tumors. Adv Immunol 2006;90: 51-81.

15. Kerkar SP, Restifo NP. Cellular constituents of immune escape within the tumor microenvironment. Cancer Res 2012;72:3125-3130.

16. Aerts JG, Hegmans JP. Tumor-specific cytotoxic T cells are crucial for efficacy of immunomodulatory antibodies in patients with lung cancer. Cancer Res 2013;73:2381-2388.

17. Mellman I, Coukos G, Dranoff G. Cance immunotherapy comes of age. Nature 2011;480 480-489.

18. Pardoll DM. The blockade of immune checkpoints in cancer immunotherapy. Nat Rev Cancer 2012;12:252-264.

19. Flies DB, Sandler BJ, Sznol M et al. Blockade of the B7-H1/PD-1 pathway for cancer immunotherapy. Yale J Biol Med 2011;84:409-421.

20. Romagné $F$, André $P$, Spee $P$ et al. Preclinical characterization of 1-7F9, a novel human anti-KIR receptor therapeutic antibody that augments natural killer-mediated killing of tumor cells. Blood 2009;114:2667-2677

21. Sakaguchi S, Wing K, Onishi $Y$ et al. Regulatory T cells: How do they suppress immune responses? Int Immunol 2009;21:1105-1111.

22. Whiteside TL. The role of death receptor ligands in shaping tumor microenvironment. Immunol Invest 2007;36:25-46.

23. Gajewski TF, Woo SR, Zha $Y$ et al. Cancer immunotherapy strategies based on overcoming barriers within the tumor microenvironment. Curr Opin Immunol 2013;25:268-276.

24. Lawrence MS, Stojanov $P$, Polak $P$ et al. Mutational heterogeneity in cancer and the search for new cancer-associated genes. Nature 2013;499: 214-218.

25. Gajewski TF. Cancer immunotherapy. Mol Oncol 2012;6:242-250.

26. Kirkwood JM, Butterfield $L H$, Tarhini AA et al. Immunotherapy of cancer in 2012. CA Cancer J Clin 2012;62:309-335.

27. Palena C, Schlom J. Vaccines against human carcinomas: Strategies to improve antitumor immune responses. J Biomed Biotechnol 2010; 380697.

28. Kantoff PW, Higano CS, Shore ND et al. Sipuleucel-T immunotherapy for castrationresistant prostate cancer. N Engl J Med 2010; 363:411-422.

29. Cheever MA, Higano CS. PROVENGE (sipuleucel-T) in prostate cancer: The first FDA-approved therapeutic cancer vaccine. Clin Cancer Res 2011;17: 3520-3526.

30. Rosenberg SA, Yang JC, Sherry RM et al. Durable complete responses in heavily pretreated patients with metastatic melanoma using T-cell transfer immunotherapy. Clin Cancer Res 2011;17: 4550-4557.

31. ClinicalTrials.gov. Available at http://clinicaltrials. gov. Accessed July 9, 2014

32. Melero I, Hervas-Stubbs $S$, Glennie $M$ et al. Immunostimulatory monoclonal antibodies for cancer therapy. Nat Rev Cancer 2007;7:95-106.

33. Hodi FS, O'Day SJ, McDermott DF et al. Improved survival with ipilimumab in patients with metastatic melanoma. N Engl J Med 2010;363: 711-723.

34. Robert C, Thomas L, Bondarenko I et al. Ipilimumab plus dacarbazine for previously untreated metastatic melanoma. N Engl J Med 2011 364:2517-2526

35. Ribas A, Camacho LH, Lopez-Berestein $\mathrm{G}$ et al. Antitumor activity in melanoma and anti-self responses in a phase I trial with the anti-cytotoxic T lymphocyte-associated antigen 4 monoclonal antibody CP-675,206. J Clin Oncol 2005;23:89688977.

36. Ribas A, Kefford R, Marshall MA et al. Phase II randomized clinical trial comparing tremelimumab with standard-of-care chemotherapy in patients with advanced melanoma. J Clin Oncol 2013;31: 616-622.

37. Eggermont AM, Chiarion-Sileni V, Grob JJ et al Ipilimumab versus placebo after complete resection of stage III melanoma: Initial efficacy and safety results from the EORTC 18071 phase III trial. J Clin Oncol 2014;32(suppl 5):LBA9008a.

38. Lynch TJ, Bondarenko I, Luft A et al. Ipilimumab in combination with paclitaxel and carboplatin as first-line treatment in stage IIIB/IV non-small-cell lung cancer: Results from a randomized, doubleblind, multicenter phase II study. J Clin Oncol 2012; 30:2046-2054.

39. Reck M, Bondarenko I, Luft A et al. Ipilimumab in combination with paclitaxel and carboplatin as first-line therapy in extensive-disease-small-cel lung cancer: Results from a randomized, doubleblind, multicenter phase 2 trial. Ann Oncol 2013;24: 75-83.

40. Wolchok JD, Hoos A, O'Day S et al. Guidelines for the evaluation of immune therapy activity in solid tumors: Immune-related response criteria. Clin Cancer Res 2009;15:7412-7420.

41. Kwon ED, Drake CG, Scher HI et al. Ipilimumab versus placebo after radiotherapy in patients with metastatic castration-resistant prostate cancer that had progressed after docetaxel chemotherapy (CA184-043): A multicentre, randomised, doubleblind, phase 3 trial. Lancet Oncol 2014;15:700-712.

42. Hamid O, Robert C, Daud A et al. Safety and tumor responses with lambrolizumab (anti-PD-1) in melanoma. N Engl J Med 2013;369:134-144.

43. Ribas A, Hodi FS, Kefford R et al. Efficacy and safety of the anti-PD-1 monoclonal antibody MK3475 in 411 patients (pts) with melanoma (MEL). $J$ Clin Oncol 2014;32(suppl 5):LBA9000^a.

44. Rizvi NR, Garon EB, Patnaik A et al. Safety and clinical activity of MK-3475 as initial therapy in patients with advanced non-small cell lung cancer (NSCLC). J Clin Oncol 2014;32(suppl 5):8007a.

45. Garon EB, Leighl NB, Rizvi NA et al. Safety and clinical activity of MK-3475 in previously treated patients (pts) with non-small cell lung cancer (NSCLC). J Clin Oncol 2014;32(suppl 5):8020a.

46. Seiwert TY, Burtness B, Weiss J et al. A phase Ib study of MK-3475 in patients with human papillomavirus (HPV)-associated and non-HPV-associated head and neck (H/N) cancer. J Clin Oncol 2014;32 (suppl 5):6011a.

47. Hodi FS, Topalian SL, Brahmer JR et al. Survival and long-term safety in patients (pts) with advanced solid tumors receiving nivolumab (anti-PD-1; BMS936558; ONO-4538) [abstract 880]. Presented at: European Cancer Congress; September 27-October 1, 2013; Amsterdam, The Netherlands.

48. Antonia SJ, Brahmer JR, Gettinger $S N$ et al Nivolumab (anti-PD-1; BMS-936558, ONO-4538) in combination with platinum-based doublet chemotherapy (PT-DC) in advanced non-small cell lung cancer (NSCLC). J Clin Oncol 2014;32(suppl 5): 8113a.

49. Rizvi NA, Chow LQM, Borghaei $\mathrm{H}$ et al. Safety and response with nivolumab (anti-PD-1; BMS936558, ONO-4538) plus erlotinib in patients (pts) with epidermal growth factor receptor mutant (EGFR MT) advanced NSCLC. J Clin Oncol 2014;32 (suppl 5):8022a.

50. Antonia SJ, Gettinger SN, Chow LQM et al. Nivolumab (anti-PD-1; BMS-936558, ONO-4538) and ipilimumab in first-line NSCLC: Interim phase results. J Clin Oncol 2014;32(suppl 5):8023a.

51. Ansell SM, Lesokhin AM, Borrello I et al. PD-1 blockade with nivolumab in relapsed or refractory Hodgkin's lymphoma. N Engl J Med 2015;372:311319.

52. Lesokhin AM, Ansell SM, Armand $P$ et al. Preliminary results of a phase I study of nivolumab (BMS-936558) in patients with relapsed or refractory lymphoid malignancies [abstract 291] Presented at: 56th ASH Annual Meeting and Exposition; December 6-9, 2014; San Francisco CA USA.

53. Atkins MB, Kudchadkar RR, Sznol M et al. Phase 2 , multicenter, safety and efficacy study of pidilizumab in patients with metastatic melanoma. J Clin Oncol 2014;32(suppl 5):9001a.

54. Soria JC, Cruz C, Bahleda R et al. Clinical activity, safety and biomarkers of PD-L1 blockade in nonsmall cell lung cancer (NSCLC): Additional analyses from a clinical study of the engineered antibody MPDL3280A (anti-PDL1) [abstract 3408]. Presented at: European Cancer Congress; September 27October 1, 2013; Amsterdam, The Netherlands.

55. Powles T, Eder JP, Fine GD et al. MPDL3280A (anti-PD-L1) treatment leads to clinical activity in metastatic bladder cancer. Nature 2014;515:558562.

56. Chen DS, Irving BA, Hodi FS. Molecular pathways: Next-generation immunotherapy-inhibiting programmed death-ligand 1 and programmed death-1. Clin Cancer Res 2012;18:6580-6587.

57. Taube JM, Klein A, Brahmer JR et al. Association of PD-1, PD-1 ligands, and other features of the tumor immune microenvironment with response to anti-PD-1 therapy. Clin Cancer Res 2014;20:50645074.

58. Kefford R, Ribas A, Hamid $O$ et al. Clinical efficacy and correlation with tumor PD-L1 expression in patients (pts) with melanoma (MEL) treated with the anti-PD-1 monoclonal antibody MK-3475. Clin Oncol 2014;32(suppl 5):3005^a.

59. Robert C, Long GV, Brady B et al. Nivolumab in previously untreated melanoma without BRAF mutation. N Engl J Med 2015;372:320-330.

60. Herbst RS, Soria JC, Kowanetz $M$ et al. Predictive correlates of response to the anti-PD-L1 antibody MPDL3280A in cancer patients. Nature 2014:515:563-567. 
61. Brignone C, Gutierrez M, Mefti F et al. First-line chemoimmunotherapy in metastatic breast carcinoma: Combination of paclitaxel and IMP321 (LAG3lg) enhances immune responses and antitumor activity. J TransI Med 2010;8:71.

62. Brignone C, Escudier B, Grygar C et al. A phase I pharmacokinetic and biological correlative study of IMP321, a novel MHC class II agonist, in patients with advanced renal cell carcinoma. Clin Cancer Res 2009;15:6225-6231.

63. Vinay DS, Kwon BS. Immunotherapy of cancer with 4-1BB. Mol Cancer Ther 2012;11:1062-1070.

64. Segal NH, Gopal AK, Bhatia Set al. A phase 1 study of PF-05082566 (anti-4-1BB) in patients with advanced cancer. J Clin Oncol 2014;32(suppl 5):3007a.

65. Curti BD, Kovacsovics-Bankowski M, Morris N et al. OX40 is a potent immune-stimulating target in late-stage cancer patients. Cancer Res 2013;73: 7189-7198.

66. Beatty GL, Chiorean EG, Fishman MP et al. CD40 agonists alter tumor stroma and show efficacy against pancreatic carcinoma in mice and humans. Science 2011;331:1612-1616.

67. Purdy AK, Campbell KS. Natural killer cells and cancer: Regulation by the killer cell Ig-like receptors (KIR). Cancer Biol Ther 2009;8:2211-2220.

68. Kohrt HE, Thielens A, Marabelle A et al. AntiKIR antibody enhancement of anti-lymphoma activity of natural killer cells as monotherapy and in combination with anti-CD20 antibodies. Blood 2014;123:678-686.

69. Benson DM Jr., Bakan CE, Zhang $S$ et al. IPH2101, a novel anti-inhibitory KIR antibody, and lenalidomide combine to enhance the natural killer cell versus multiple myeloma effect. Blood 2011; 118:6387-6391.

70. Uyttenhove C, Pilotte L, Théate let al. Evidence for a tumoral immune resistance mechanism based on tryptophan degradation by indoleamine 2,3dioxygenase. Nat Med 2003;9:1269-1274.

71. Liu X, Shin N, Koblish HK et al. Selective inhibition of IDO1 effectively regulates mediators of antitumor immunity. Blood 2010;115: 3520-3530.

72. McDermott D, Haanen J, Chen TT et al. Efficacy and safety of ipilimumab in metastatic melanoma patients surviving more than 2 years following treatment in a phase III trial (MDX010-20). Ann Oncol 2013;24:2694-2698.

73. Maio M, Grob JJ, Aamdal S et al. Five-year survival rates for treatment-naive patients with advanced melanoma who received ipilimumab plus dacarbazine in a phase III trial. J Clin Oncol 2015;33: 1191-1196.

74. Schadendorf D, Hodi F, Robert C et al. Pooled analysis of long-term survival data from phase II and phase III trials of ipilimumab in unresectable or metastatic melanoma. J Clin Oncol 2015 [Epub ahead of print].
75. Brahmer JR, Horn L, Gandhi L et al. Nivolumab (anti-PD-1, BMS-936558, ONO-4538) in patients (pts) with advanced non-small-cell lung cancer (NSCLC): Survival and clinical activity by subgroup analysis. J Clin Oncol 2014;32(suppl 5): $8112^{\wedge} \mathrm{a}$

76. Hodi FS, Sznol M, Kluger HM et al. Long-term survival of ipilimumab-naive patients (pts) with advanced melanoma (MEL) treated with nivolumab (anti-PD-1, BMS-936558, ONO-4538) in a phase I trial. J Clin Oncol 2014;32(suppl 5):9002a.

77. Postow MA, Harding J, Wolchok JD. Targeting immune checkpoints: Releasing the restraints on anti-tumor immunity for patients with melanoma. Cancer J 2012;18:153-159.

78. Amos SM, Duong CP, Westwood JA et al. Autoimmunity associated with immunotherapy of cancer. Blood 2011;118:499-509.

79. Di Giacomo AM, Biagioli M, Maio M. The emerging toxicity profiles of anti-CTLA-4 antibodies across clinical indications. Semin Oncol 2010;37: 499-507.

80. Fecher LA, Agarwala SS, Hodi FS et al. Ipilimumab and its toxicities: A multidisciplinary approach. The Oncologist 2013;18:733-743.

81. Chow LQ. Exploring novel immune-related toxicities and endpoints with immune-checkpoint inhibitors in non-small cell lung cancer. Am Soc Clin Oncol Educ Book 10.1200/EdBook_AM.2013.33. e280.

82. Ledezma B, Heng A. Real-world impact of education: Treating patients with ipilimumab in a community practice setting. Cancer Manag Res 2013;6:5-14.

83. Topalian SL, Sznol M, Brahmer JR et al. Nivolumab (anti-PD-1; BMS-936558; ONO-4538) in patients with advanced solid tumors: Survival and long-term safety in a phase I trial. J Clin Oncol 2013; 31(suppl 15):3002a.

84. Wolchok JD, Kluger $\mathrm{H}$, Callahan MK et al. Nivolumab plus ipilimumab in advanced melanoma. N Engl J Med 2013;369:122-133.

85. Sznol M, Kluger HM, Callahan MK et al. Survival, response duration, and activity by BRAF mutation (MT) status of nivolumab (NIVO, anti-PD1, BMS-936558, ONO-4538) and ipilimumab (IPI) concurrent therapy in advanced melanoma (MEL). $J$ Clin Oncol 2014;32(suppl 5):LBA9003^a.

86. Kershaw MH, Devaud C, John LB et al. Enhancing immunotherapy using chemotherapy and radiation to modify the tumor microenvironment. Oncolmmunology 2013;2:e25962.

87. Formenti SC, Demaria S. Combining radiotherapy and cancer immunotherapy: A paradigm shift. J Natl Cancer Inst 2013;105:256-265.

88. Ménard C, Martin F, Apetoh L et al. Cancer chemotherapy: Not only a direct cytotoxic effect, but also an adjuvant for antitumor immunity. Cancer Immunol Immunother 2008;57:1579-1587.
89. Moschella F, Proietti E, Capone I et al. Combination strategies for enhancing the efficacy of immunotherapy in cancer patients. Ann N Y Acad Sci 2010;1194:169-178.

90. Vanneman M, Dranoff G. Combining immunotherapy and targeted therapies in cancer treatment. Nat Rev Cancer 2012;12:237-251.

91. Drake CG. Combination immunotherapy approaches. Ann Oncol 2012;23:viii41-viii46.

92. Kroemer G, Galluzzi L, Kepp O et al. Immunogenic cell death in cancer therapy. Annu Rev Immunol 2013;31:51-72.

93. Barker CA, Postow MA. Combinations of radiation therapy and immunotherapy for melanoma: A review of clinical outcomes. Int J Radiat Oncol Biol Phys 2014;88:986-997.

94. Ribas A, Hodi FS, Callahan M et al. Hepatotoxicity with combination of vemurafenib and ipilimumab. N Engl J Med 2013;368:1365-1366

95. Johnson DB, Wallender EK, Cohen DN et al. Severe cutaneous and neurologic toxicity in melanoma patients during vemurafenib administration following anti-PD-1 therapy. Cancer Immunol Res 2013;1:373-377.

96. Ascierto PA, Simeone E, Giannarelli $D$ et al. Sequencing of BRAF inhibitors and ipilimumab in patients with metastatic melanoma: A possible algorithm for clinical use. J Transl Med 2012;10: 107.

97. Ascierto PA, Simeone $E$, Sileni $V C$ et al. Sequential treatment with ipilimumab and BRAF inhibitors in patients with metastatic melanoma: Data from the Italian cohort of the ipilimumab expanded access program. Cancer Invest 2014;32: 144-149.

98. Hoos A. Evolution of end points for cancer immunotherapy trials. Ann Oncol 2012;23:viii47viii52.

99. Topalian SL, Hodi FS, Brahmer JR et al. Safety, activity, and immune correlates of anti-PD-1 antibody in cancer. N Engl J Med 2012;366:2443-2454.

100. Pennock GK, Waterfield W, Wolchok JD. Patient responses to ipilimumab, a novel immunopotentiator for metastatic melanoma: How different are these from conventional treatment responses? Am J Clin Oncol 2012;35:606611.

101. Hodi FS, Ribas A, Daud A et al. Evaluation of immune-related response criteria (irRC) in patients (pts) with advanced melanoma (MEL) treated with the anti-PD-1 monoclonal antibody MK-3475. J Clin Oncol 2014;32(suppl 5):3006a.

102. Hamid O, Sosman JA, Lawrence DP et al. Clinical activity, safety, and biomarkers of MPDL3280A, an engineered PD-L1 antibody in patients with locally advanced or metastatic melanoma (mM). J Clin Oncol 2013;31(suppl 15): 9010a. 